\title{
Adaptação intercultural para português europeu do questionário "Conocimiento del Paciente sobre sus Medicamentos” (CPM-ES-ES)
}

\author{
Cross-cultural adaptation to the European Portuguese \\ of the questionnaire "Patient Knowledge about their Medications" \\ (CPM-ES-ES)
}

Joaquín Salmerón Rubio ${ }^{1}$

Paula Iglésias-Ferreira ${ }^{2}$

Pilar García Delgado ${ }^{1}$

Henrique Mateus-Santos ${ }^{2}$

Fernando Martínez-Martínez ${ }^{1}$

\footnotetext{
${ }^{1}$ Grupo de Investigación en Atención Farmacéutica (CTS-131), Facultad de Farmacia, Universidad de Granada. Calle Real de Cartuja 36, Campus de Cartuja. 18.012 Granada España.

farjoaquin@gmail.com ${ }^{2}$ Grupo de Investigação em Cuidados Farmacêuticos (GICUF), Universidade Lusófona de Humanidades e Tecnologias (Lisboa).
}

\begin{abstract}
The scope of this work is to conduct the cross-cultural adaptation from Spanish to European Portuguese of a questionnaire to measure the degree of "Patient Knowledge about their Medications" (CPM-ES-ES). A method based on six steps was applied: 1. Translation into Portuguese, 2. Elaboration of the first consensus version in Portuguese; 3.Back-translation into Spanish; 4. Elaboration of the second consensus version (cultural equivalency); 5 . Conducting the pre-test; 6 . Evaluation of the overall results. A cross-culturally adapted questionnaire in European Portuguese that measures the degree of "Patient Knowledge about their Medications" is proposed. The pretest confirmation obtained 100\% agreement with the corrected version of the second consensus version after pre-testing. The methodology selected made it possible to cross-culturally adapt the Spanish version of the CPM-ES-ES questionnaire to the Portuguese version. Further studies should demonstrate the equivalence of the psychometric properties of the cross-cultural translation into Portuguese with the original version.
\end{abstract}

Key words Questionnaires, Cross-cultural adaptation, Patients, Medicine, Knowledge
Resumo O objetivo deste trabalho é realizar a adaptação intercultural do espanhol para português europeu do questionário para medir o grau do "Conocimiento del Paciente sobre sus Medicamentos" (CPM-ES-ES). Aplicou-se um método baseado em seis etapas: 1. Tradução para português; 2. Preparação da primeira versão de consenso em português; 3. Retroversão para espanhol; 4. Preparação da segunda versão (equivalência cultural) de consenso; 5. Realização do pré-teste; 6. Avaliação dos resultados globais. Propõe-se um questionário adaptado culturalmente em português europeu que mede o grau do "conhecimento do doente sobre os seus medicamentos". O préteste de confirmação obteve $100 \%$ de concordância com a segunda versão de Consenso corrigida após o pré-teste. A metodologia selecionada permitiu adaptar culturalmente a versão em espanhol do questionário CPM-ES-ES para uma versão em português. Estudos adicionais devem demonstrar a equivalência das propriedades psicométricas da tradução intercultural em português do questionário com a versão original.

Palavras-chave Questionários, Adaptação intercultural, Pacientes, Medicamento, Conhecimento 


\section{Introdução}

As falhas da farmacoterapia são uma realidade. A morbimortalidade relacionada com o uso dos medicamentos tem uma prevalência tão elevada que constitui um problema de saúde pública ${ }^{1-4}$.

O conhecimento do paciente sobre a sua medicação é um dos determinantes para o uso racional do medicamento, o que contribui, embora não garanta, para o aumento dos resultados positivos associados à medicação ${ }^{5}$.

Se o paciente não tem a informação correta sobre cada um dos aspetos básicos do processo de uso do medicamento pode utilizá-lo de modo inadequado, com a possibilidade de originar um resultado negativo ${ }^{6,7}$.

García-Delgado et al. ${ }^{8,9}$ definem "grau de conhecimento do paciente sobre os seus medicamentos" como "o conjunto da informação adquirida pelo paciente sobre o seu medicamento necessária para um uso correto do mesmo, a qual inclui o objetivo terapêutico (indicação e efetividade), o processo de uso (posologia, esquema terapêutico, forma de administração e duração do tratamento), a segurança (efeitos adversos, precauções, contraindicações e interações) e a sua conservação.

Existem diferentes ferramentas para avaliar aspetos concretos dos medicamentos, assim como questionários validados para o conhecimento de doenças concretas ${ }^{10,11}$. Contudo, o único questionário publicado para medir, de modo global, o conhecimento do paciente sobre os seus medicamentos é o validado por García-Delgado et al. ${ }^{9}$, "Conocimiento del Paciente sobre sus Medicamentos" (CPM-ES-ES). Este questionário, elaborado em espanhol europeu, demonstrou propriedades psicométricas robustas que justificam, por si só, a sua utilização em estudos realizados em outras culturas ou línguas: a validade de conteúdo foi verificada através de uma exaustiva pesquisa bibliográfica e com três técnicas qualitativas (painel de peritos, tormenta de ideias e Delphi) e a validade do constructo foi calculada através da análise fatorial dos componentes principais que evidenciaram a existência de 4 dimensões que explicam $66,99 \%$ da variância total; a consistência interna foi avaliada através cálculo do Coeficiente de Alpha de Cronbach que foi de 0,677 $(0,60$ a 0,72 nas diferentes dimensões do questionário); a equivalência do questionário, avaliada pelo grau de concordância interobservadores (Coeficiente Kappa = 0,99); e a estabilidade avaliada pelo Coeficiente de Correlação Intraclasse (CCI) que foi de 0,745 (IC 95\%:0,49-0,87) ${ }^{9}$.
Por ser um questionário com um caráter geral, o CPM-ES-ES pode aplicar-se a qualquer paciente em tratamento com qualquer tipo de medicamento e que apresente uma patologia qualquer 9 .

Historicamente, a adaptação de instrumentos elaborados numa outra língua submetia-se a traduções literais do original ou, excecionalmente, a comparações literais de este com versões retrovertidas ${ }^{12}$. Atualmente, considera-se que não deve existir apenas uma tradução linguística, mas sim uma adaptação cultural que permita garantir a validade do conteúdo do instrumento sob o ponto de vista conceptual ${ }^{13}$.

Apesar do desenvolvimento na área da tradução linguística nos últimos anos, ainda não existe um consenso global sobre qual a melhor estratégia para realizar uma adaptação intercultural, existindo na literatura diferentes propostas metodológicas para a realização deste processo, estando ainda, o modo de chegar à equivalência entre a versão adaptada interculturalmente e a original em discussã $0^{13-32}$.

O objetivo deste trabalho é realizar a adaptação intercultural do espanhol para português europeu do questionário para medir o grau do "Conocimiento del Paciente sobre sus Medicamentos" (CPM-ES-ES).

\section{Método}

Obteve-se a autorização dos autores da versão original do questionário CPM-ES-ES para realizar a adaptação intercultural para português europeu.

O questionário concebido e validado por Garcia-Delgado et al. em espanhol europeu é constituído por 23 itens, sendo que apenas 11 integram a escala que mede o conhecimento do medicamento. Estes 11 itens abordam a indicação, a posologia, o esquema terapêutico, a duração do tratamento, a forma de administração, as precauções, os efeitos adversos, as contraindicações, a efetividade, as interações e a conservação, refletindo quatro dimensões: "objetivo terapêutico" (item 6 e 14), "processo de uso dos medicamentos" (itens $7,8,9,10$ ), "segurança" (itens 11, 12, $13,15)$ e "conservação" (item 16) $)^{8-11,30,33}$.

Após estudar as técnicas de adaptação intercultural publicadas, elegeu-se a metodologia desenhada por Guillemin e por Beaton ${ }^{34,35} \mathrm{com}$ as adaptações necessárias, a este tipo de tradução, propostas por Arribas ${ }^{36}$. Para verificar a equivalência intercultural entre as duas versões utili- 
zou-se uma escala analógica visual para a comissão de peritos avaliar o processo de adaptação intercultural $^{31,37}$.

O processo de adaptação intercultural desenvolveu-se em seis etapas (Figura 1).

Etapa 1 - Tradução para português: Foram realizadas duas traduções (T1 e T2) do questionário CPM-ES-ES original do espanhol europeu para o português europeu por dois farmacêuticos comunitários bilingues, isto é, que tinham o português como língua materna e com domínio acreditado do idioma original do instrumento (espanhol), que realizaram, cada um deles, de forma independente, uma primeira tradução do questionário para o português. A um dos tradu- tores (T1), disponibilizou-se informação das características do questionário e dos parâmetros que teria que avaliar, assim como instruções para que a terminologia fosse simples. O outro tradutor (T2) não recebeu qualquer tipo de recomendações.

Etapa 2 - Preparação da primeira versão de consenso em português (Consenso 1): Os dois tradutores juntamente com um grupo de peritos formados por professores de farmácia prática da Universidade Lusófona (Lisboa), membros do Grupo de Investigação em Cuidados Farmacêuticos (GICUF), reviram ambas as traduções e realizaram uma reconciliação dos itens problemáticos com o objetivo de criar a primeira tra-

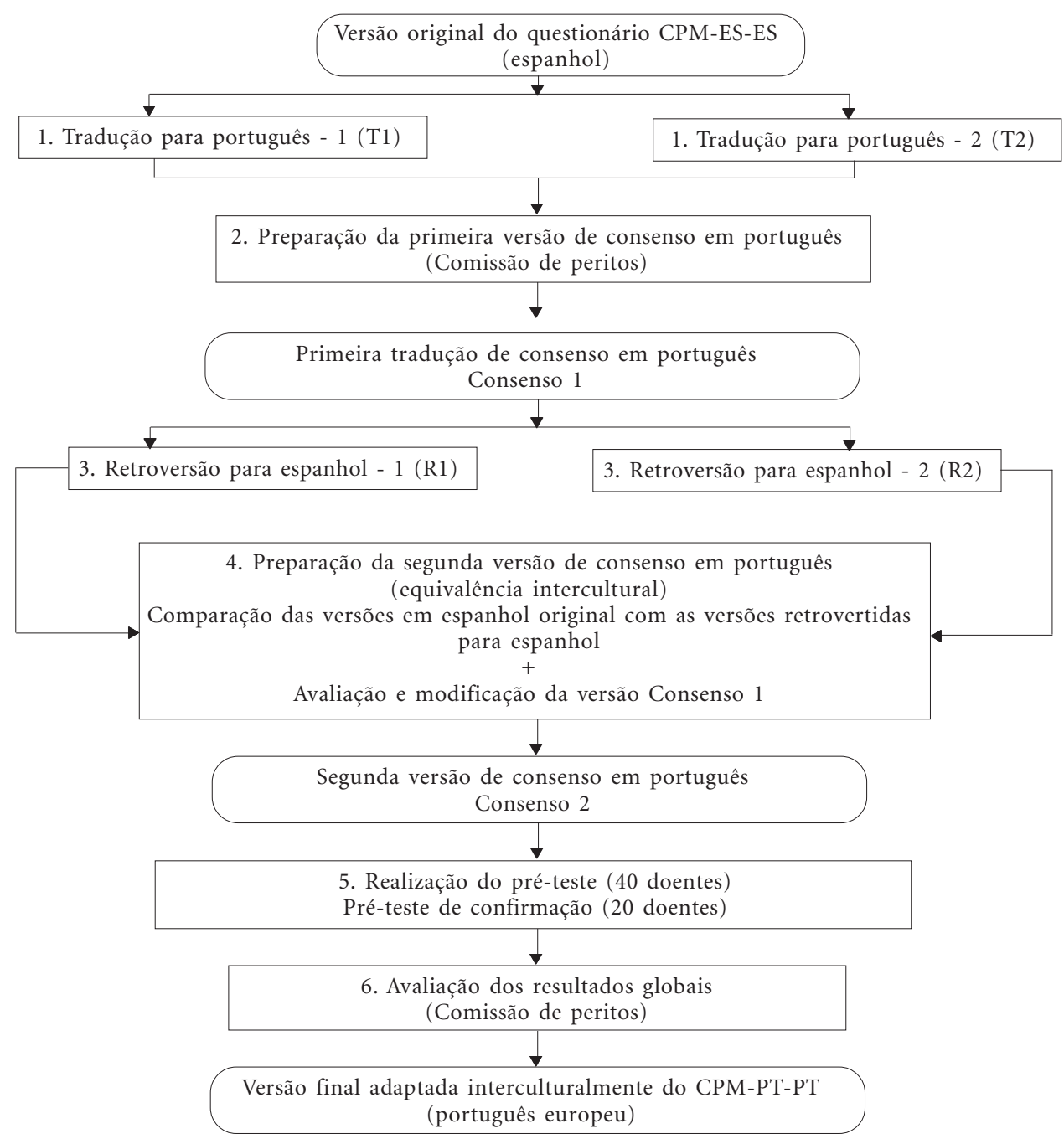

Figura 1. Fluxograma do processo de tradução intercultural do CPM-ES-ES para português europeu. 
dução de consenso traduzida em português europeu (Consenso 1).

Etapa 3 - Retroversão para espanhol: A tradução Consenso 1 foi submetida a duas retroversões independentes (R1 e R2) para espanhol por dois tradutores com língua materna espanhola e domínio acreditado em português. Os tradutores desconheciam a versão original do questionário e trabalharam em separado.

Etapa 4 - Preparação da segunda versão de consenso (equivalência intercultural) (Consenso 2): Pretende-se com esta etapa avaliar o grau da equivalência intercultural e preparar uma versão de consenso que tenha o mesmo significado (equivalência intercultural) na população portuguesa que o instrumento original na população espanhola. Para avaliar a equivalência intercultural, consideraram-se as quatro áreas de equivalência: semântica (as palavras terem o mesmo significado), idiomática (existir equivalência de provérbios ou expressões coloquiais), experiencial ou cultural, ou seja, da experiência dos termos empregados, a equivalência das situações descritas ou retratadas na versão original devem adaptar-se ao contexto da cultura alvo em que se realiza, com vocabulário simples, direto e conceptual (existe homogeneidade de conceitos entre as culturas?) e a equivalência conceptual, a equivalência dos conceitos e dos acontecimentos experienciados pelas pessoas na cultura alvo ${ }^{34,35}$. Embora possa haver diferenças entre o grau de compreensão na leitura entre os questionários na língua espanhola e portuguesa estabelece-se como requisito obrigatório, que seja compreensível na leitura por qualquer pessoa que fale a língua portuguesa que tenha o nível de instrução correspondente ao sexto ano de educação primária ${ }^{37}$.

Foi realizada a avaliação (da concordância entre a versão original do questionário e a retroversão) da equivalência intercultural com recurso à medição do significado referencial (A1) e do significado geral (A2) propostas por Streiner e Nor$\operatorname{man}^{37}$, cujo objetivo é avaliar as diferenças literais, isto é, do significado das palavras e de sentido (conceitos), respetivamente, entre a versão original e as respetivas retroversões (R1 e R2).

Para cada aspeto de equivalência intercultural avaliado, foram usados formulários específicos e a medição foi realizada com recurso a uma escala analógica visual.

Primeiro, avaliou-se o significado referencial que consiste na equivalência literal entre cada palavra do instrumento original e das suas retroversões (R1 e R2). Presume-se que, se o significado referencial é o mesmo no original e na res- petiva retroversão existe uma correspondência literal entre estes.

Para avaliar o significado referencial ponderou-se a equivalência dos itens do questionário original e dos das retroversões (R1 e R2) correspondentes de forma continuada com valores que vão de 0 a $100 \%$, sendo $0 \%$ a equivalência mínima e $100 \%$ a máxima (ou concordância total). Estabelecendo como valores de máxima equivalência $100 \%, 80-89$ quase equivalentes e $<80 \%$, não equivalentes ${ }^{37}$.

O segundo aspeto apreciado foi o significado geral de cada pergunta, instrução ou opção de resposta do instrumento captado nas duas retroversões em comparação com o instrumento original em espanhol. Esta correspondência transcende a literalidade de termos ou frases, incluindo aspetos mais subtis, como, por exemplo, o impacto que estas têm no contexto cultural da população-alvo. Nesse sentido, interessa avaliar a pertinência e a aceitabilidade do estilo empregado ou o uso específico de uma palavra, escolhida dentre uma gama de termos similares. Esta apreciação é importante porque a correspondência literal de um termo não implica, necessariamente, que a mesma reação emocional ou afetiva seja evocada em diferentes culturas. Assim, é indispensável uma sintonia cuidada que alcance também uma correspondência de perceção e de impacto.

Para avaliar o significado geral (A2) comparou-se os itens do questionário original em espanhol com cada item de cada uma das retroversões (R1 e R2) utilizando uma classificação com quatro níveis: inalterado (IN), pouco alterado (PA), muito alterado (MA) ou completamente alterado $(\mathrm{CA})^{37}$.

Para cada item foi utilizado um formulário no qual os pares das retroversões (R1 e R2) foram confrontados com o questionário original.

Elaborou-se um segundo relatório correspondente ao processo de análise intercultural que apresentava: uma análise individual de cada pergunta do questionário, das instruções do mesmo, das diferentes respostas do questionário sobre a primeira versão portuguesa e a justificação em cada caso.

A comissão de peritos com base nos resultados de concordância obtidos para cada um dos itens elaborou uma segunda versão de tradução de consenso (Consenso 2) com base nos itens da tradução T1 e T2 que obtiveram maior concordância na avaliação da respetiva retroversão contra a versão original do instrumento.

Etapa 5 - Realização do pré-teste: O pré-teste pretendeu avaliar como funciona o questionário 
Consenso 2 e se seriam necessárias alterações antes da sua aplicação em estudos no terreno.

Selecionou-se um grupo reduzido de 40 indivíduos, nos quais estiveram representados os diversos segmentos que compõem a amostra (Tabela 1).

A recolha dos questionários foi realizada numa farmácia de Lisboa durante uma semana.

Os questionários foram preenchidos por entrevista direta farmacêutico-paciente, por um único entrevistador, previamente preparado para o efeito, que realizou os seguintes passos:

1. Apresentação do projeto de investigação ao paciente selecionado e do consentimento informado para participar no estudo;

2. Leitura integral das perguntas do questionário por parte do entrevistador e transcrição das respostas dadas pelos pacientes. Realizou-se uma única entrevista para apenas um medicamento de cada paciente.
No final, administrou-se um questionário, por entrevista direta, de avaliação cognitiva, com 10 questões para identificar quais as perguntas que originaram mais dificuldades, para determinar as razões destas dificuldades e ainda para registar as soluções propostas para melhorar a clareza das perguntas ${ }^{38}$. Todos os comentários foram registados. Elaborou-se um relatório final desta etapa.

Pelo facto de algumas palavras terem demonstrado ser de difícil compreensão pelos entrevistados, após as retificações, foi elaborado um novo questionário que foi submetido a um pré-teste de confirmação, por entrevista direta farmacêutico-paciente, a 20 pacientes, seguindo a mesma metodologia utlizada no pré-teste.

Etapa 6 - Avaliação dos resultados globais (preparação da versão final do questionário para medir o grau do conhecimento que os pacientes têm sobre os seus medicamentos em português

Tabela 1. Características sociodemográficas dos pacientes incluídos no pré-teste e no pré-teste de confirmação.

\begin{tabular}{|c|c|c|c|}
\hline Variáveis independentes & Segmentação das variáveis & $\begin{array}{l}\text { Pré-teste } \\
\quad \mathbf{x}(\%)\end{array}$ & $\begin{array}{c}\text { Pré-teste de confirmação } \\
\mathbf{x}(\%)\end{array}$ \\
\hline No pacientes & $\mathrm{n}$ & 40 & 20 \\
\hline \multirow[t]{2}{*}{ Género } & Homens & $11(27,5)$ & $6(30)$ \\
\hline & Mulheres & $29(72,5)$ & $14(70)$ \\
\hline Idade (média) & Anos & 60,36 & 57,95 \\
\hline \multirow[t]{4}{*}{ Nível de estudos } & Sem estudos & $1(2,5)$ & $2(10)$ \\
\hline & Estudos primários & $30(75)$ & $8(40)$ \\
\hline & Estudos secundários & $7(17,5)$ & $7(35)$ \\
\hline & Estudos universitários & $2(5)$ & $3(15)$ \\
\hline \multirow{3}{*}{$\begin{array}{l}\text { Número de medicamentos } \\
\text { que toma }\end{array}$} & Monoterapia & $5(12,5)$ & $2(10)$ \\
\hline & De 2 a 4 & $10(25)$ & $6(30)$ \\
\hline & Polimedicação ( $>5$ medicamentos) & $25(62,5)$ & $12(60)$ \\
\hline \multirow[t]{3}{*}{ Prescritor do medicamento } & Médico & $37(92,5)$ & $20(100)$ \\
\hline & Farmacêutico & $2(5)$ & 0 \\
\hline & Automedicação & $1(2,5)$ & 0 \\
\hline \multirow{5}{*}{$\begin{array}{l}\text { Tempo de uso do } \\
\text { medicamento }\end{array}$} & Primeira prescrição & $8(20)$ & $3(15)$ \\
\hline & De 0,5 a 6 meses & $5(12,5)$ & $2(10)$ \\
\hline & De 7 a 12 meses & $7(17,5)$ & $3(15)$ \\
\hline & De 13 a 24 meses & $5(12,5)$ & $3(15)$ \\
\hline & Mais de 24 meses & $15(37,5)$ & $9(45)$ \\
\hline \multirow{3}{*}{$\begin{array}{l}\text { Importância para o } \\
\text { paciente do seu problema } \\
\text { de saúde }\end{array}$} & Pouco & $9(22,5)$ & $4(20)$ \\
\hline & Regular & $8(20)$ & $9(45)$ \\
\hline & Bastante & $23(57,5)$ & $7(35)$ \\
\hline
\end{tabular}


europeu): Nesta fase, integraram a comissão de peritos os tradutores, os tradutores que fizeram a retroversão, o investigador principal, os professores da Universidade Lusófona, um professor da Universidade de Granada e os autores do questionário original. Reuniram-se e de um modo definitivo verificaram os relatórios e o cumprimento de cada etapa assim como a análise individual de cada pergunta do questionário e das instruções.

Registaram-se as dificuldades detetadas durante o pré-teste, avaliou-se o grau de concordância entre as traduções Consenso 1 e Consenso 2 , as modificações realizadas sobre a tradução Consenso 2 em português na versão do pré-teste de confirmação e a justificação para cada caso, optando-se pela versão dos itens com maior grau de concordância dando origem à versão final do questionário para medir o grau de Conhecimento dos Pacientes sobre os seus Medicamentos (CPM-PT-PT) adaptado interculturalmente para o português europeu.

\section{Resultados}

\section{Avaliação da equivalência intercultural}

Foi realizada a avaliação da concordância para o significado referencial e para o significado geral de cada item de cada uma das retroversões (R1 e R2) contra a versão original do instrumento CPM-ES-ES em espanhol (Tabela 2).

A partir dos resultados obtidos em cada item a comissão de peritos criou uma segunda tradução de consenso (Consenso 2) que adotou o item da tradução (T1 ou T2) que alcançou maior concordância neste processo.

Título: Optou-se pelo termo "doente" por a retroversão R2 ter alcançado uma concordância de $100 \%$ e IN, respetivamente para o significado referencial e para o significado geral, mantendose a versão da tradução T2. A comissão de peritos considerou que a palavra doente, parecia ser mais geral e integradora. A avaliação da concordância entre a tradução Consenso 1 e Consenso 2 foi total.

Instruções: na avaliação do significado geral ficaram inalteradas as duas traduções no estudo comparativo com as suas respetivas retroversões, assim como a versão de consenso elaborada pela comissão de peritos.

Dados: Hora de início do questionário, dia de realização, nome da farmácia e número do questionário. Foi obtida a concordância total na retroversão e na avaliação da tradução de Consenso 1 e Consenso 2.

Questionário (itens 1 a 23): Foi elaborada uma tradução Consenso 2 optando pelos itens da tradução que alcançaram uma maior concordância para o significado referencial e para significado geral na etapa 4, isto é durante a tradução intercultural.

$\mathrm{Na}$ avaliação da tradução Consenso 1 e Consenso 2 a maioria dos itens apresentou uma percentagem de concordância do significado referencial de 100\% (catorze itens), 98\% (dois itens), 95\% (cinco itens) e apenas dois itens obtiveram 90\%. Na avaliação do significado geral, dezanove itens obtiveram um nível inalterado (IN), quatro itens obtiveram um nível pouco alterado (PA) e nenhum item alcançou um nível de muito alterado (MA) ou completamente alterado (CA).

A tradução Consenso 2 foi submetida a um pré-teste e por terem surgido problemas de entendimento foram introduzidas alterações que motivaram a realização de um pré-teste de confirmação.

\section{Resultados do pré-teste e pré-teste de confirmação}

A amostra do pré-teste foi composta por 40 pacientes, dos quais, 29 eram mulheres (Tabela 1). A idade média da amostra foi de 60,3 anos, com um mínimo de 23 anos e um máximo de 95 anos. A maioria (30 indivíduos) concluiu apenas o ensino primário. Cada paciente foi questionado apenas sobre um medicamento. $\mathrm{O}$ médico foi o prescritor em 37 dos medicamentos sobre os quais se mediu o conhecimento.

Dos 40 pacientes, 23 atribuíam bastante importância ao seu problema de saúde. É importante destacar que se tratou de um início de tratamento apenas em 8 casos e que 35 dos 40 pacientes entrevistados tomavam mais que um medicamento.

Todos os entrevistados concordaram que o questionário não incluía nenhuma pergunta em excesso nem desnecessária.

Verificou-se durante o pré-teste que algumas perguntas eram de difícil interpretação por parte da população em estudo, pelo que foram introduzidas alterações no questionário: 1) o termo "nível de estudos" foi alterado para "nível de instrução" 2) o termo "regular" que não era entendido pela população portuguesa, foi substituído por "mais ou menos", por ser mais comum 3) a pergunta referente ao item 16 "Como deve conservar o seu medicamento?” foi alterada para "que 
Tabela 2. Resultados das avaliações da concordância entre as etapas da adaptação intercultural

\begin{tabular}{|c|c|c|c|c|c|c|c|c|}
\hline \multirow{3}{*}{$\begin{array}{l}\text { Instrumento } \\
\text { Original } \\
\text { (espanhol) } \\
\text { Pergunta/item }\end{array}$} & \multicolumn{4}{|c|}{$\begin{array}{l}\text { Tradução intercultural } \\
\text { (Etapa 1-2-3) } \\
\text { Comissão de Peritos }\end{array}$} & \multirow{2}{*}{\multicolumn{2}{|c|}{$\begin{array}{c}\text { Avaliação final } \\
\text { (Etapa 4-5) } \\
\text { Comissão de Peritos } \\
\text { Avaliação da } \\
\text { concordância entre } \\
\text { C1 e o C2 }\end{array}$}} & \multirow{2}{*}{\multicolumn{2}{|c|}{$\begin{array}{c}\begin{array}{c}\text { Avaliação final } \\
\text { (Etapa 5-6) }\end{array} \\
\text { Comissão de Peritos } \\
\begin{array}{c}\text { Avaliação da } \\
\text { concordância entre } \\
\text { C2 e após pré-teste }\end{array}\end{array}$}} \\
\hline & \multicolumn{2}{|c|}{$\begin{array}{c}\text { Avaliação da } \\
\text { concordância entre } \\
\text { CPM-ES-ES e a R1 }\end{array}$} & \multicolumn{2}{|c|}{$\begin{array}{l}\text { Avaliação da } \\
\text { concordância entre } \\
\text { CPM-ES-ES e a R2 }\end{array}$} & & & & \\
\hline & A1 (\%) & A2 & A1 (\%) & A2 & A1 (\%) & A2 & A1 (\%) & A2 \\
\hline Título & 98 & PA & 100 & IN & 100 & IN & 100 & IN \\
\hline Instruções & 95 & IN & 95 & IN & 95 & IN & 100 & IN \\
\hline Dados & 100 & IN & 100 & IN & 100 & IN & 100 & IN \\
\hline Item 1 & 90 & $\mathrm{PA}$ & 95 & PA & 95 & PA & 100 & IN \\
\hline Item 2 & 98 & PA & 100 & IN & 100 & IN & 100 & IN \\
\hline Item 3 & 100 & IN & 100 & IN & 100 & IN & 100 & IN \\
\hline Item 4 & 100 & IN & 100 & IN & 100 & IN & 100 & IN \\
\hline Item 5 & 100 & IN & 100 & IN & 100 & IN & 100 & IN \\
\hline Item $6^{*}$ & 100 & IN & 100 & IN & 100 & IN & 100 & IN \\
\hline Item $7^{*}$ & 100 & IN & 100 & IN & 100 & IN & 100 & IN \\
\hline Item $8^{*}$ & 95 & PA & 90 & $\mathrm{PA}$ & 95 & $\mathrm{PA}$ & 100 & IN \\
\hline Item $9^{*}$ & 100 & IN & 100 & IN & 100 & IN & 100 & IN \\
\hline Item $10^{*}$ & 95 & $\mathrm{PA}$ & 95 & PA & 95 & $\mathrm{PA}$ & 100 & IN \\
\hline Item $11^{*}$ & 98 & PA & 98 & PA & 98 & $\mathrm{PA}$ & 100 & IN \\
\hline Item $12^{*}$ & 100 & IN & 100 & IN & 100 & IN & 100 & IN \\
\hline Item $13^{*}$ & 100 & IN & 100 & IN & 100 & IN & 100 & IN \\
\hline Item $14^{*}$ & 100 & IN & 100 & IN & 100 & IN & 100 & IN \\
\hline Item $15^{*}$ & 95 & $\mathrm{PA}$ & 100 & IN & 95 & IN & 100 & IN \\
\hline Item $16^{*}$ & 90 & IN & 100 & IN & 90 & IN & 90 & $\mathrm{PA}$ \\
\hline Item 17 & 90 & IN & 100 & IN & 90 & IN & 90 & $\mathrm{PA}$ \\
\hline Item 18 & 100 & IN & 100 & IN & 100 & IN & 100 & IN \\
\hline Item 19 & 100 & IN & 100 & IN & 100 & IN & 100 & IN \\
\hline Item 20 & 100 & IN & 100 & IN & 100 & IN & 100 & IN \\
\hline Item 21 & 100 & IN & 98 & IN & 98 & IN & 100 & IN \\
\hline Item 22 & 100 & IN & 100 & IN & 100 & IN & 100 & IN \\
\hline Item 23 & 95 & IN & 100 & IN & 95 & IN & 95 & $\mathrm{PA}$ \\
\hline
\end{tabular}

$\mathrm{A} 1=$ Avaliação percentual de concordância do significado referencial. A2= Avaliação qualitativa da concordância do significado geral segundo as categorias: $\mathrm{IN}=$ Inalterado; $\mathrm{PA}=$ Pouco alterado; $\mathrm{MA}=$ Muito alterado; $\mathrm{CA}=\mathrm{Completamente}$ alterado. ${ }^{*}$ Itens que medem o grau do conhecimento sobre o medicamento.

cuidados deve ter para manter em bom estado de conservação o seu medicamento?", após consulta a um perito em língua portuguesa para garantir que não se adulterava o sentido original.

Para garantir que todas as alterações realizadas sobre o questionário tinham entendimento adequado foi realizado um segundo pré-teste a 20 pacientes, dos quais 14 eram mulheres. A idade média da amostra foi de 58 anos. A maioria ${ }^{8}$ concluiu apenas o ensino primário. Cada paciente foi questionado apenas sobre um medicamento. $\mathrm{O}$ médico foi o prescritor de todos os medicamentos.

Destes 20 pacientes, 7 atribuíam bastante importância ao seu problema de saúde. É importante destacar que se tratou de um início de tratamento apenas em 3 casos e que 18 dos 20 pacientes entrevistados tomavam mais que um medicamento.

As alterações realizadas e testadas no pré-teste de confirmação demonstraram ter um entendimento total.

Por último, verificaram-se todos os relatórios, o cumprimento de cada etapa, assim como a análise individual de cada pergunta do questionário e das instruções. Registaram-se todas as dificuldades detetadas durante o trabalho de campo, as modificações realizadas na segunda versão em português e a justificação para cada caso.

O resultado deste processo é o questionário CPM-PT-PT (Quadro 1) adaptado interculturalmente ao português europeu que estará pron- 
to para ser submetido ao processo de avaliação das suas propriedades psicométricas em estudos posteriores.

\section{Discussão}

Selecionou-se o questionário CPM-ES-ES de Garcia-Delgado et al. ${ }^{9}$ por ser um instrumento desenvolvido e testado no contexto da farmácia comunitária para medir o grau de conhecimento dos pacientes sobre os seus medicamentos. As propriedades psicométricas do questionário CPM-ESES demonstraram uma fiabilidade considerada adequada (Coeficiente Alpha de Cronbach de 0,68 ), um grau de concordância elevado (Kappa $=0,99)$ e uma estabilidade boa $(\mathrm{CCI}=0,77)$, motivos pelos quais selecionámos este questionário para ser submetido ao processo de tradução intercultural de espanhol para português (europeu), apesar de serem necessários estudos adicionais que demonstrem a validade do instrumento?.

A maioria dos questionários publicados estão na língua inglesa e foram desenvolvidos para culturas anglo-saxónicas. $\mathrm{Na}$ área da saúde, os que foram mais traduzidos para outras línguas, para uso noutros países, são na sua maioria, questionários destinados a avaliar qualidade de vida relacionada com a saúde.

As diferentes fontes bibliográficas consultadas sugerem a necessidade de se adaptar interculturalmente para o português o instrumento original em espanhol para medir o grau de conhecimento que têm os pacientes sobre os seus medicamentos, embora ambas as línguas sejam latinas e muito semelhantes.

O objetivo do processo de adaptação intercultural é elaborar um instrumento adaptado às pessoas objeto da investigação, quer dizer, à população portuguesa e que seja equivalente ao instrumento original, evitando-se assim adaptações posteriores para as diversas subculturas e, elaborando-se, tanto quanto possível, um instrumento que possa ser utilizado pela maior parte da população.

A tradução intercultural transforma uma mensagem de um idioma noutro. O processo é complexo e exige uma técnica metódica e sistemática que permita: a) transferir dados do idioma de origem ao idioma destino, b) manter ou estabelecer a equivalência intercultural.
A realização do pré-teste realizado com a tradução Consenso 2 permitiu identificar palavras que, embora tenham sido aceites pelos investigadores, tradutores e pela comissão de peritos, demonstraram ter um difícil entendimento na população alvo do questionário. Este foi o motivo pelo qual a comissão de revisão optou pelas palavras/expressões "manter em bom estado de conservação" no item 16, "mais ou menos" no item 17 e "nível de instrução" no item 23, dando origem a uma tradução final (tradução intercultural) do questionário CPM-PT-PT que ao ser submetida a um pré-teste de confirmação obteve $100 \%$ de concordância em todos os itens com a versão Consenso 2 corrigida após o pré-teste.

A técnica utlizada para avaliar as alterações realizadas em cada etapa permitiu que a comissão de peritos tivesse uma perceção global sobre o grau de alterações em cada etapa quanto ao significado referencial e ao significado geral e introduzir as alterações necessárias com vista à obtenção de uma versão final do questionário.

A equivalência intercultural consegue-se mediante a interpretação, que vai mais além da tradução palavra a palavra para explicar o significado dos conceitos, utilizando temos compreensíveis e as normas gramaticais do idioma destino.

O método de adaptação intercultural incluiu orientações para obter a equivalência semântica, idiomática, experiencial e conceitual das traduções ${ }^{34,35}$. Seu objetivo é facilitar o processo de adaptação intercultural para preservar a sensibilidade do instrumento da cultura de chegada.

A origem comum do português e do espanhol acentua a necessidade de fazer uma tradução intercultural para que não se cometam erros de tradução, pois a aparente similaridade entre as duas línguas pode esconder muitas diferenças ${ }^{39}$.

A existência de palavras conhecidas como 'falsos cognatos', 'falsos amigos' ou palavras de tradução enganosa fazem com que esta tarefa deva ser realizada por tradutores experientes e por profissionais entendidos na área a que se refere $o$ documento. A tradução por etapas adiciona qualidade ao trabalho e a participação multidisciplinar dos intervenientes no processo garante que a tradução obtida seja um documento, no que diz respeito aos conceitos, equivalente ao original ${ }^{40}$.

A adaptação intercultural permite que a versão final seja um documento de trabalho onde estão minimizadas as diferenças de significado entre o texto inicial e o final. 
Quadro 1. Perguntas incluídas na versão original do questionário CPT-ES-ES e perguntas na versão final da tradução intercultural do questionário CPT-PT-PT.

\begin{tabular}{|c|c|c|}
\hline Pergunta & $\begin{array}{l}\text { Versão original em espanhol } \\
\text { do CPM-ES-ES }\end{array}$ & $\begin{array}{l}\text { Versão adaptada em português europeu } \\
\text { do CPM-PT-PT }\end{array}$ \\
\hline Item 1 & $\begin{array}{l}\text { ¿Es para usted este medicamento?¿Es para } \\
\text { alguien que está a su cuidado? }\end{array}$ & $\begin{array}{l}\text { Este medicamento é para si? Se não, é para } \\
\text { alguém que está ao seu cuidado? }\end{array}$ \\
\hline Item 2 & ¿Cómo se llama este medicamento? & $\begin{array}{l}\text { Em relação a este medicamento por favor } \\
\text { registe, Nome comercial, Princípio ativo, } \\
\text { Forma farmacêutica. }\end{array}$ \\
\hline Item 3 & ¿Conoce el nombre del medicamento? & $\begin{array}{l}\text { Como se chama este medicamento? O } \\
\text { doente conhece o nome do medicamento? }\end{array}$ \\
\hline Item 4 & $\begin{array}{l}\text { ¿Desde cuando está tomando/utilizando } \\
\text { este medicamento? }\end{array}$ & $\begin{array}{l}\text { Desde quando está a tomar/utilizar este } \\
\text { medicamento? }\end{array}$ \\
\hline Item 5 & $\begin{array}{l}\text { ¿Cuántos medicamentos está tomando } \\
\text { además de éste? }\end{array}$ & $\begin{array}{l}\text { Quantos medicamentos está a tomar para } \\
\text { além deste? }\end{array}$ \\
\hline Item $6^{*}$ & $\begin{array}{l}\text { ¿Para qué tiene que tomar este } \\
\text { medicamento? }\end{array}$ & $\begin{array}{l}\text { Para que tem que tomar/utilizar este } \\
\text { medicamento? }\end{array}$ \\
\hline Item $7^{*}$ & $\begin{array}{l}\text { ¿Qué cantidad debe tomar/utilizar de este } \\
\text { medicamento? }\end{array}$ & $\begin{array}{l}\text { Quanto deve tomar/utilizar deste } \\
\text { medicamento? }\end{array}$ \\
\hline Item $8^{*}$ & $\begin{array}{l}\text { ¿Cada cuánto tiene que tomar/utilizar este } \\
\text { medicamento? }\end{array}$ & $\begin{array}{l}\text { Quando tem que tomar/utilizar este } \\
\text { medicamento? }\end{array}$ \\
\hline Item $9^{*}$ & $\begin{array}{l}\text { ¿Hasta cuando tiene que tomar/utilizar este } \\
\text { medicamento? }\end{array}$ & $\begin{array}{l}\text { Até quando tem que tomar/utilizar este } \\
\text { medicamento? }\end{array}$ \\
\hline Item $10^{*}$ & $\begin{array}{l}\text { ¿Cómo debe tomar/utilizar este } \\
\text { medicamento? }\end{array}$ & $\begin{array}{l}\text { Como debe tomar/utilizar este } \\
\text { medicamento? }\end{array}$ \\
\hline Item $11^{*}$ & $\begin{array}{l}\text { ¿Ha de tener alguna precaución cuando } \\
\text { toma/utiliza este medicamento? }\end{array}$ & $\begin{array}{l}\text { Deve ter alguma precaução quando toma/ } \\
\text { utiliza este medicamento? }\end{array}$ \\
\hline Item $12^{*}$ & $\begin{array}{l}\text { ¿Qué efectos adversos conoce usted de este } \\
\text { medicamento? }\end{array}$ & $\begin{array}{l}\text { Que efeitos adversos conhece deste } \\
\text { medicamento? }\end{array}$ \\
\hline Item $13^{*}$ & $\begin{array}{l}\text { ¿Ante qué problema de salud o situación } \\
\text { especial no debe tomar/utilizar este } \\
\text { medicamento? }\end{array}$ & $\begin{array}{l}\text { Em que situações ou casos não deve tomar/ } \\
\text { utilizar este medicamento? }\end{array}$ \\
\hline Item $14^{*}$ & $\begin{array}{l}\text { ¿Cómo sabe si el medicamento le hace } \\
\text { efecto? }\end{array}$ & $\begin{array}{l}\text { Como é que sabe se o medicamento lhe faz } \\
\text { efeito? }\end{array}$ \\
\hline Item $15^{*}$ & $\begin{array}{l}\text { ¿Qué medicamentos o alimentos debe evitar } \\
\text { tomar mientras use este medicamento? }\end{array}$ & $\begin{array}{l}\text { Que medicamentos ou alimentos debe } \\
\text { evitar enquanto usa este medicamento? }\end{array}$ \\
\hline Item $16^{*}$ & ¿Cómo debe conservar su medicamento? & $\begin{array}{l}\text { Que cuidados deve ter para manter em bom } \\
\text { estado de conservação o seu medicamento? }\end{array}$ \\
\hline Item $17^{*}$ & $\begin{array}{l}\text { ¿El problema de salud para el que toma este } \\
\text { medicamento, le preocupa...bastante, } \\
\text { regular ó poco? }\end{array}$ & $\begin{array}{l}\text { O problema de saúde para o qual toma este } \\
\text { medicamento preocupa-o: muito, mais ou } \\
\text { menos ou pouco? }\end{array}$ \\
\hline Item 18 & $\begin{array}{l}\text { ¿Quién le digo que tomara/utilizara este } \\
\text { medicamento? }\end{array}$ & $\begin{array}{l}\text { Quem lhe disse para tomar/utilizar este } \\
\text { medicamento? }\end{array}$ \\
\hline Item 19 & Género & Género \\
\hline Item 20 & Edad & Idade \\
\hline Item 21 & Profesión & Profissão \\
\hline Item 22 & País de origen & País de origem \\
\hline Item 23 & Nivel de estudios & Nível de instrução \\
\hline
\end{tabular}

*(item 6 ao 17) Itens que medem o grau de conhecimento sobre os medicamentos. 


\section{Conclusões}

Instrumentos elaborados numa língua original necessitam de um processo sistemático de adaptação intercultural para poderem ser utilizados em realidades socioculturais distintas. A metodologia selecionada permitiu adaptar interculturalmente a versão espanhola do questionário "Conocimiento del Paciente sobre sus Medicamentos" (CPM-ES-ES) para a versão portuguesa "Conhecimento do doente sobre os seus medicamentos" (CPM-PT-PT).

Os resultados obtidos demonstram que a versão portuguesa e espanhola são conceptualmente equivalentes.

Estudos adicionais devem demonstrar a equivalência das propriedades psicométricas (fiabilidade e validade) da versão portuguesa para que possa ser utilizado em investigação e serviços de cuidados farmacêuticos.
A utilização deste questionário contribui para determinar se existe uma necessidade de informação por parte dos pacientes e permite identificar os aspetos concretos onde existe falta de informação. A aplicação desta ferramenta permite medir o grau de conhecimento dos portugueses sobre os medicamentos que usam. Também avaliar e classificar se a informação que detêm é suficiente ou insuficiente para garantir um processo de uso correto do (s) medicamento (s). Consequentemente, permite estimar e justificar o emprego de meios e de pessoas qualificadas para implementarem programas de educação/orientação terapêutica adequados e adaptados às necessidades dos pacientes. $\mathrm{O}$ resultado da aplicação deste instrumento permitirá ainda identificar oportunidades de melhoria para a prática farmacêutica, tais como, entre outras, desenvolver ações que promovam a eficácia do processo de transmissão da informação personalizada sobre os medicamentos que cada paciente necessita.

\section{Colaboradores}

JS Rubio, P Iglésias-Ferreira, PG Delgado, H Mateus-Santos e F Martínez-Martínez participaram igualmente de todas as etapas de elaboração do artigo. 


\section{Referências}

1. Johnson JA, Bootman JL. Drug-related morbidity and mortality. A cost-of-illness Model. Arch Intern Med. 1995 9;155(18):1949-56.

2. Ernst FR, Grizzle AJ. Drug-related morbidity and mortality: updating the cost-of-illness model. $J$ Am Pharm Assoc 2001; 41(2):192-199.

3. Climente M, Quintana I, Martinez G, Atienza A, Jiménez NV. Prevalencia y características de la morbilidad relacionada con los medicamentos como causa de ingreso hospitalario. Aten Farm 2001; 3(1):9-22.

4. Baena MI, Faus MJ, Fajardo PC, Luque FM, Sierra F, Martinez-Olmos J, Cabrera A, Fernandez-Llimos F, Martinez-Martinez F, Jiménez J, Zarzuelo A. Medicine-related problems resulting in emergency department visits. Eur J Clin Pharmacol 2006; 62(5):387-393.

5. Cegala DJ, Marinelli T, Post D. The effects of patient communication skills training on compliance. Arch Fam Med 2000; 9(1):57-64.

6. DeVries TPGM, Henning RH, Hogerzeil HV, Fresle DA. Guide to good prescribing. Geneva: WHO/Action programme on essential drugs; 1994.

7. DeVries TPGM. Presenting clinical pharmacology and therapeutics: A problem based approach for choosing and prescribing drugs. Br J Clin Pharmacol 1993; 35:581-586.

8. Garcia-Delgado P. Conocimiento de los pacientes sobre sus medicamentos. Granada: Universidade de Granada; 2008.

9. García-Delgado P, Gastelurrutia MA, Baena MI, Fisac F, Martínez-Martínez F. Validación de un cuestionario para medir el conocimiento de los pacientes sobre sus medicamentos. Aten Primaria 2009; 41(12):661-669.

10. Iglesias JCA, Rodriguez NFA, Perez JAF. Validación de un cuestionario de conocimientos sobre hipercolesterolemia en la farmacia comunitaria. Seguim Farmacot 2005; 3(4):189-196.

11. Rodriguez C, Sossa MP. Validación de un cuestionario de conocimientos acerca del asma entre padres o tutores de niños asmáticos. Arch Bronconeumol 2005; 41(8):419-424.

12. Chassany O, Sagnier P, Marquis P, Fulleton S, Aaronson N. for the European Regulatory Issues on Quality of Life Assessment (ERIQA) Group. Patient-reported outcomes: the example of Health-Related Quality of Life - a European guidance document for the improved integration of Health-Related Quality of Life assessment in the drug approval process. DIA J 2001; 36(1):913-923.

13. Bullinger M, Power MJ, Aaronson NK. Creating and evaluating cross-cultural instruments. In: Spilker B, editor. Quality of Life and Pharmacoeconomics in Clinical Trials. $2^{\text {nd }}$ Edition. New York: Lippincott-Raven Publishers; 1996.

14. Bullinger M, Alonso J, Apolone G, Leplege A, Sullivan M, Wood-Dauphinee S, Gandek B, Wagner A, Aaronson N, Bech P, Fukuhara S, Kaasa S, Ware JE Junior. Translating health status questionnaires and evaluating their quality: the IQOLA Project approach. International Quality of Life Assessment. J Clin Epidemiol 1998; 51(11):913-923.
15. Ware JE, Jr., Keller SD, Gandek B, Brazier JE, Sullivan M. Evaluating translations of health status questionnaires. Methods from the IQOLA project. International Quality of Life Assessment. Int J Technol Assess Health Care 1995; 11(3):525-551.

16. Acquadro C, Conway K, Giroudet C, Mear I. Linguistic Validation Manual for Patient-Reported Outcomes (PRO) Instruments. Lyon: Mapi Research Institut; 2004.

17. Mear I. Health-Related Quality of Life and PatientReported Outcomes: Scientific and Useful Criteria. In: Chassany O, Caulin C, editors. Difficulties of international clinical trials: cultural adaptation of quality of life questionnaires. Paris: Springer; 2002.

18. Moraes CL, Hasselmann MH, Reichenheim ME. Adaptação transcultural para o português do instrumento "Revised Conflict Tactics Scales (CTS2)" utilizado para identificar violência entre casais. Cad Saude Publica 2002; 18(1):163-176.

19. Anderson RT, Aaronson NK, Wilkin D. Critical review of the international assessments of health related quality of life. Qual Life Res 1993; 2:369-395.

20. Anderson RT, Aaronson NK, Bullinger M, WL M. A review of the progress towards developing health related quality of life instruments for international clinical studies and outcomes research. Pharmacoeconomics 1996; 10:336-334.

21. Schmidt S, Bullinger M. Current issues in crosscultural quality of life instrument development. Arch Phys Med Rehabil 2003; 84(Supl. 2):S29-S34.

22. Guyatt GH. The philosophy of health-related quality of life translation. Qual Life Res 1993; 2(6):461465.

23. Hunt SM, Bhopal R. Self report in clinical and epidemiological studies with non-English speakers: the challenge of language and culture. J Epidemiol Community Health 2004; 58(7):618-622.

24. Hunt SM, Alonso J, Bucquet D, Niero M, Wiklund I, McKenna S. European Group for Health Measurement and Quality of Life Assessment. Cross-cultural adaptation of health measures. Health Policy 1991; 19(1):33-44.

25. Hunt SM. Cross-cultural issues in the use of sociomedical indicators. Health Policy 1986; 6(2):149-158.

26. Bowden A, Fox-Rushby JA. A systematic and critical review ofthe process of translation and adaptation of generic health-related quality of life measures in Africa, Asia, Eastern Europe, the Middle East, South America. Soc Sci \& Med 2003; 57(7):12891306.

27. Falcao DM, Ciconelli RM, Ferraz MB. Translation and cultural adaptation of quality of life questionnaires: an evaluation of methodology. J Rheumatol 2003; 30(2):379-385.

28. Leplège A, Verdier A. The adaptation of health status measures: methodological aspects of the translation procedure. In: Shumaker SA, Berzon R, editors. The International Assessment of Health-Related Quality of Life: Theory, Translation, Measurement \& Analysis. Oxford: Rapid Communications; 1995.

29. Maneesriwongul W, Dixon JK. Instrument translation process: a methods review. J Adv Nurs 2004; 48(2):175-186. 
30. Badía X, Baró E. Cuestionarios de salud en España y su uso en atención primaria. Aten Primaria 2001; 28(5):349-356.

31. Holanda WTG, Lima MLCd, Figueiro JN. Adaptação transcultural de um instrumento de avaliação do handicap auditivo para portadores de perda auditiva induzida pelo ruído ocupacional. Cien Saude Colet 2011; 16(Supl. 1):755-767.

32. da Franca C, Colares V. Validação do National College Health Risk Behavior Survey para utilização com universitários brasileiros. Cien Saude Colet 2010; 15(Supl. 1):1209-1215.

33. Pita Fernandéz S, Pértegas Díaz S. La fiabilidad de las mediciones clínicas: el análisis de concordancia para variables numericas. [sítio en Internet]. [cited 200916 Nov]. Available from: http://www. fisterra.com/mbe/investiga/conc_numerica/conc_ numerica.asp.

34. Guillemin F, Bombardier C, Beaton D. Cross-cultural adaptation of health-related quality of life measures: literature review and proposed guidelines. J Clin Epidemiol 1993; 46(12):1417-1432.

35. Beaton DE, Bombardier C, Guillemin F, Ferraz MB. Guidelines for the process of cross-cultural adaptation of self-report measures. Spine 2000; 25(24): 3186-3191.

36. Arribas A. Adaptación transcultural de instrumentos: Guía para el proceso de validación de instrumentos tipo encuestas. Rev Científica de la $A M B B$ 2006; 16(3):74-82.
37. Streiner DL, Norman GR. Consistencia, validez y fiabilidad en las escalas de medida de la salud (Adaptado por Grau-Fibla G). In: Streiner DL, Norman GR, editors. Health measurement scales: a practical guide to their development and use. $2^{\text {nd }}$ Edition. New York: Oxford University Press; 1992.

38. Secherist L, Fay TL, Zaidi SMH. Problems of translation in cross-cultural research. Journal of CrossCultural Psychology 1972; 3(1):41-56.

39. Santos H, Iglésias-Ferreira P, Fernández-Llimós F, Faus MJ, Rodrigues LM. Segundo Consenso de Granada sobre Problemas Relacionados com Medicamentos - Tradução Intercultural de Espanhol para Português (europeu). Acta Médica Portuguesa 2004; 17(1):59-66.

40. Iglésias-Ferreira P, Santos HJ, Fernández-Llimós F, Fontes E, Leal M, Monteiro C. Traducción y Validación del "Pharmacy Services Questionnaire" al portugués (europeo). Seguim Farmacoter 2005; $1(3): 43-56$.

Artigo apresentado em 20/08/2012

Aprovado em 30/11/2012

Versão final apresentada em 16/12/2012 